\title{
Manifiesto en Tiempos de Pandemia: Por una Educación Crítica, Intergeneracional, Sostenible y Comunitaria
}

\author{
A Manifest in a Time of Pandemic: For a Communitarian, \\ Sustainable, Intergenerational and Critical Education
}

\author{
Edgar Iglesias 1 \\ Javier González-Patiño ${ }^{2}$ \\ José Luis Lalueza ${ }^{3}$ \\ Moisés Esteban-Guitart 1 \\ ${ }^{1}$ Universitat de Girona, España \\ ${ }^{2}$ Universidad Autónoma de Madrid, España \\ ${ }^{3}$ Universitat Autònoma de Barcelona, España
}

\begin{abstract}
La actual emergencia derivada de la pandemia del coronavirus nos recuerda que compartimos un mismo desafío en tanto que seres humanos. Recientemente, se ha considerado la pandemia Covid-19 una experiencia colectiva y traumática ("world perezhivanie”), caracterizada por una crisis sistémica que impacta no solo en nuestra salud, sino también en nuestra economía, política, orden social y, por supuesto, en la educación. Esta situación nos impide postergar más la necesidad aún pendiente de (re)imaginar críticamente el sentido de la educación en el siglo XXI. En este artículo teórico, de carácter propositivo y deliberativo, proponemos dos principios para superar tres sesgos habituales en nuestra compresión del aprendizaje y del hecho educativo. A partir de dichas consideraciones, se describe e ilustra lo que para nosotros significa una educación intergeneracional, personalizada, compartida, conectada y sostenible, basada en una cultura y práctica de los afectos y el acompañamiento, que permita consolidar procesos de mejora y transformación educativa.
\end{abstract}

Descriptores: Justicia social; Pedagogías disruptivas; Acompañamiento; Equidad; Pandemia; Educación post-Covid-19.

The emergency resulting from the coronavirus pandemic reminds us that, as human beings, we share a common challenge. Recently, it has been suggested that Covid-19 pandemic involve a "world perezhivanie", a collective and traumatic "lived experience", characterized by a profound crisis that affects not only our health, but also our economic, social, political and educational systems. In the light of this situation, we need to critically re-imagine education in $21^{\text {st }}$ century. In this theoretical paper, we propose two principles designed to overcome three common biases in our understanding of learning and education. From these assumptions, we describe and illustrate the meaning and practice behind an inter-generational, personalized, shared, connected and sustainable education, based on a culture and praxis that is caring and involved, that allows us to consolidate processes of educational transformation and improvement.

Keywords: Social justice; Pedagogies of disruption; Caring; Equity; Pandemic; Post-Covid-19 education.

*Contacto: edgar.iglesiasv@udg.edu

ISSN: 2254-3139

www.rinace.net/riejs/

revistas.uam.es/riejs
Recibido: $\quad 15$ de mayo 2020

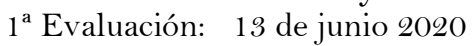

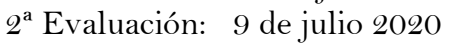

Aceptado: 14 de julio 2020 


\section{Introducción: La otra educación que era posible ahora es necesaria}

A pesar de que no podemos saber lo que sucederá en unos años, ni tan siquiera en unas semanas, debido a la incertidumbre, riesgo y liquidez de un mundo que se nos escapa entre los dedos como el agua (Bauman, 2006); sí que debemos imaginar-proyectar, analizar, discutir y reflexionar sobre algunas limitaciones, también oportunidades, que atraviesan nuestra historia contemporánea. En este sentido, el Covid-19, lejos de aparecer exclusivamente como un fenómeno médico-sanitario, se erige como una realidad poliédrica de un gran impacto económico, social, mediático, político y, por supuesto, educativo (Cervantes Holguín y Gutiérrez Sandoval, 2020; Von Braun, Zamagni y Sorondo, 2020).

La adopción masiva de la enseñanza a distancia de manera precipitada - definida como enseñanza remota de emergencia (Bozkurt y Sharma, 2020)- ha permitido que afloren algunas contradicciones de los sistemas educativos, las más evidentes en las denominadas "escuelas de alta complejidad" en entornos sociales con alto riesgo de exclusión social, donde se ha confirmado la brecha social que obstaculiza la ya de por sí complicada digitalización de la enseñanza (Álvarez, Gardyn, Iardelevsky y Rebello, 2020; Murillo y Duk, 2020), llegando a cuestionarse el propio derecho a la educación (Ruiz, 2020). Una brecha de equipamiento tecnológico (viviendas sin wifi, sin ordenador o compartido para todos los convivientes, con un único dispositivo móvil para toda la familia sujeto a un exiguo plan de datos); habitacional (inadecuación de la vivienda por carencia de espacio, masificación o falta de intimidad); educativa (escaso capital cultural de los padres en el ámbito de la educación formal y/o de los medios digitales) y lingüística (desconocimiento de la lengua vehicular de la escuela por algunas familias, dificultades de comunicación con las maestras y maestros). Muchas voces han dado la alarma (ver, en este sentido, Cabrera, 2020), y se da por sentado que en muchos casos no sólo se "perderá el curso" sino que se corre el riesgo de una desconexión duradera, quizás definitiva, con la escuela.

Sin embargo, una aproximación solamente carencial puede conducir a una visión estigmatizadora basada en lo que la literatura se ha descrito como perspectiva del déficit en educación (Valdés, 1996; Valencia, 2010). Es decir, la vinculación de estudiantes de grupos minoritarios -o infra-representados en la cultura y práctica normativa escolar- a determinados déficits, problemas y carencias: procesos inadecuados de socialización familiar, inteligencia limitada, limitaciones de tipo cultural, carencias económicas y lingüísticas que acaban dificultando e impidiendo sus procesos de aprendizaje.

Contrariamente, más de cuatro décadas de investigación en la aproximación de los fondos de conocimiento (Llopart y Esteban-Guitart, 2018; Moll, 2019) muestra que todas las familias -más allá de su condición, origen y diversidad social, económica, cultural disponen de saberes, habilidades, relaciones y destrezas, resultado de sus trayectorias de participación en prácticas socioculturales varias (por ejemplo, prácticas productivas como el trabajo, o recreativas y comunitarias como el juego o la religión). Sin embargo, dichas competencias, fortalezas y recursos no siempre son identificados/as, reconocidos/as, legitimados/as e incorporados/as en la práctica educativa escolar, existiendo disímiles procesos de (dis)continuidades entre las culturas y prácticas 
escolares y familiares en función del grupo y clase socioeconómica prevaleciente en la práctica de la institución formal de enseñanza y aprendizaje (Poveda, 2001).

Así, se estima que el hogar -como contexto de socialización- debiera ser comprendido también como potencial fuente de recursos y aprendizaje, incluso en la situación de pandemia reciente (Ishimaru, 2020). También, como hipótesis de trabajo, que cabría corroborar empíricamente, las actividades de vinculación entre las escuelas y las familias, así como actores educativos externos varios, que en algunos casos se estima muy significativa, puede evidenciar algunas cosas que ya se sabían, que incluso formaban parte de los programas de mejora y transformación escolar, pero que ahora se han mostrado imprescindibles. A saber: la importancia de las emociones en los procesos de aprendizaje, el acompañamiento y los cuidados (Tarabini, 2020); la conveniencia de una personalización de las trayectorias de aprendizaje (Coll, Esteban-Guitart e Iglesias, 2020); O incluso, aspectos relativos a la diversificación de las formas de relación con las familias y la necesidad de su inclusión en la dinámica escolar (Moll, 2019), la importancia del trabajo en red con entidades del entorno comunitario (Rodríguez-Ruiz, ÁlvarezBlanco, Martínez-González y Epstein, 2019), el inaplazable requerimiento de incorporar lo digital como una forma de dar sentido al mundo en el que vivimos (González-Patiño y Esteban-Guitart, 2014), así como el carácter intergeneracional y colaborativo de los procesos de aprendizaje (Nasir et al., 2020; Rogoff, 2016).

En definitiva, es probable que el impacto de la pandemia y el confinamiento hayan supuesto altas dosis de estrés para el profesorado, pero también puede haber contribuido en la creación de condiciones para que los sistemas de enseñanza (las escuelas y sus entornos comunitarios) aprendan (Engeström, 2001). En cualquier caso, la acentuación de las contradicciones de la brecha social ha contribuido a evidenciar la necesidad de cambio y a identificar algunas de las claves que lo hacen posible.

Según datos oficiales de la $\mathrm{UNESCO}^{1}$ se estima que más del $70 \%$ del total de alumnos y alumnas matriculados y matriculadas, dato que representa más de 1.000.000.000 de estudiantes, en 186 contextos nacionales distintos en todo el planeta, se han visto afectados por el cierre de las escuelas, institutos y universidades en el tercer semestre del curso escolar 2019-2020. Un "déficit de realidad" (cancelación de los procesos de socialización e interacción física, por ejemplo, en los centros educativos formales, sustituidos por una mediación tecnológica para el aprendizaje remoto) que ha puesto en evidencia, entre otras cuestiones:

- Las brechas, desigualdades e injusticias educativas varias (Álvarez et al., 2020; Cabrera, 2020; Murillo y Duk, 2020), a las que anteriormente hacíamos referencia;

- La necesidad del acceso universal a internet: hoy un espacio público, también privado, de creación e intercambio identitario, económico, social, político y cultural. Un acceso que debe ir acompañado de una necesaria incorporación crítica para darle sentido a las formas en que habitamos dicho espacio. Lo que constituye para nosotros un derecho humano básico y fundamental en las actuales Sociedades Móvil-Céntricas (González-Patiño y Esteban-Guitart, 2014);

\footnotetext{
${ }^{1}$ https://es.unesco.org/covid 19/educationresponse
} 
- No obstante, y paralelamente, la importancia del acompañamiento y presencia física para promover procesos de interacción y socialización plena, así como la escuela como elemento vertebrador de procesos de igualdad y cohesión social, a pesar de sus contradicciones y efectos a veces en dirección opuesta (Tarabini, 2020);

- Así como y, por todo ello, la necesidad de acelerar los procesos de mejora, transformación y contextualización de las instituciones formales de enseñanza y aprendizaje (escuelas, institutos, universidades) ante una crisis profunda de su sentido y legitimidad que viene anunciándose en las últimas décadas (Coll, 2013) y que la reciente pandemia mundial ha contribuido a poner en evidencia.

Persiguiendo unos objetivos claros, ¿̇para qué?

Los Objetivos de Desarrollo Sostenible (ODS) de la $\mathrm{ONU}^{2}$, a pesar de ser, quizá por ello, ambiciosos y, aunque en el marco de una visión decolonial de los mismos (ColladoRuano, 2016), se presentan como un marco idóneo para situar los desafíos que atraviesan nuestras sociedades. Poner fin a la pobreza mundial en todas sus formas; garantizar una vida sana y promover el bienestar; garantizar modalidades de consumo y producción sostenibles; adoptar medidas para combatir el colapso ecológico o lograr la igualdad entre géneros, así como garantizar una educación inclusiva, equitativa y de calidad, y promover oportunidades de aprendizaje durante toda la vida para todos y todas, pueden actuar de espejo-utopía para proyectar y facilitar "pensamientos holísticos genuinos sobre futuros posibles, combinado con procesos democráticos de implicación, reflexividad y provisionalidad" (Levitas, 2013, p. xi).

Se trata, como proponen Jenkins, Peters-Lazaro y Shresthova (2020), a propósito del término "imaginación cívica", de construir colectivamente visiones para un mejor mañana, conjuntamente con la actualización de procesos de cambio, basados en la agencia cívica de las personas y, en base a alianzas de cooperación y solidaridad, también de confrontación y contestación económica, política, social y cultural.

En realidad, se observan preocupaciones, temas y prácticas convergentes entre distintos países, a pesar de sus diferencias y contradicciones, lo que apoya pensar en la posibilidad de una comunidad planetaria, inteligencia colectiva o, según Blunden (2020, p. 1), cierta "vivencia mundial" (world-perezhivanie) asociada a la pandemia: "Estoy convencido de que la actual pandemia, entendida junto con todas las diversas respuestas a la misma, tanto inmediatas como posteriores, es una experiencia que cambiará el mundo. En particular, entiendo esta experiencia como una perezhivanie.” Eso es: una experiencia personalcolectiva frente a una situación traumática que puede llevar a procesos de mejora y transformación.

Para poder transformar el mundo es necesario, efectivamente, comprenderlo (Santos, 2018). Solamente así es posible romper, para proponer, alternativas ontológicas (prácticas), epistemológicas (saberes y conocimiento) y axiológicas (valores) a las actuales condiciones sociales, culturales, políticas y educativas (Rodríguez-Romero, 2020). Consideramos que aquí se halla el sentido más profundo del espacio, la práctica y la cultura de la educación.

${ }^{2}$ https://www.un.org/sustainabledevelopment/es/objetivos-de-desarrollo-sostenible/ 
En el ámbito de la pedagogía, currículum y educación formal, ello se traduce en la propuesta, e implementación, de pedagogías disruptivas, democráticas, decoloniales, antirracistas, orientadas hacia la justicia social, comprometidas con un proceso continuo de reflexión y mejora permanente (Belavi y Murillo, 2020; Giroux, 2015; Murillo y Hernández, 2014; Rodríguez-Romero, 2020) y, por lo tanto, alejadas de las pedagogías de la indiferencia (Lingard y Keddie, 2013) que terminan por omisión, naturalizando la injusticia y desigualdad.

Por supuesto la escuela no puede ser responsable, y "vacuna", frente a los problemas mundiales. Pero sí puede propiciar, fomentar y revisar críticamente estilos de vida dañinos hacia concepciones críticas de especie más responsables e inteligentes basadas en la solidaridad, la diversidad, la empatía, la confianza mutua, la cooperación y una organización social, en definitiva, que permita la supervivencia de nuestra especie (Carbonell, 2020). Misión que no puede hacer aisladamente. La educación es contingente a una realidad social, histórica, tecnológica, económica y cultural particular; y en la medida que esta cambia, la educación está obligada a pertenecer a las condiciones de la sociedad que, a su vez, la crea. No en vano, una educación descontextualizada es una educación vacía, sin sentido.

\section{Qué relación hay entre desarrollo humano y educación}

A nuestro juicio existen tres sesgos -reduccionismos- que han caracterizado lo que comúnmente se ha entendido, y se entiende, por aprendizaje y educación (Richmond, 2018).

- Sesgo cognitivo en el aprendizaje. Énfasis en los aprendizajes intelectualesconceptuales-instrumentales (operaciones hipotético-deductivas) en detrimento de los procesos psicológicos superiores directivos como la identidad, las narrativas e imaginarios (artes, culturas, medios), las emociones y sentimientos, o los cuidados y compromisos-responsabilidades sociales. Lo que promueve un aprendizaje con significado (establecimiento de relaciones sustanciales entre las ideas, conocimientos y saberes previos con las/los nuevos), pero sin sentido (sin necesariamente valor identitario-subjetivopersonal). Retomando la visión orgánica y pedagógica desde las tres dimensiones (cabeza, corazón y manos) de Pestalozzi (1746-1827), el énfasis excesivo en los aprendizajes intelectuales o instrumentales, activa frecuentemente la cabeza, pero no necesariamente las manos (acción) ni el corazón (implicación, afectividad) (Brühlmeier, 2012). Tampoco, necesariamente, deviene en impacto y contribución social.

- Sesgo espacial del acto educativo. El discurso mayoritario asociado a los procesos de enseñanza y aprendizaje se reduce a la práctica reglada, deliberada e intencional: instituciones formales (escuelas, institutos, universidades). Sin menospreciar la importancia que tienen dichas instituciones, se las condena con la exclusividad hacia la responsabilidad del acto de aprender y enseñar. 
- Sesgo temporal del acto educativo. Los destinatarios de la enseñanza deliberada e intencional, prácticas educativas formales, son -principalmentelos niños y niñas, adolescentes y jóvenes. Se excluye de la ecuación la madurez y personas mayores; menos aún el contacto intergeneracional.

Dichas limitaciones constituyen planteamientos que han dominado, en nuestra opinión, parte de la literatura educativa contemporánea, así como el sentido común. Frente a ellas, planteamos dos contrapropuestas que en forma de principios debieran tenerse en cuenta en lo que denominamos como educación post-Covid-19.

$\mathrm{El}$ aprendizaje. Lejos de ser un fenómeno meramente cognitivo-instrumental, surge de la experiencia como proceso sostenido e inacabado de construcción identitaria-biográfica: ser un docente competente, médico, activista, madre/padre, lampista, científico, músico, ciudadano. Este planteamiento nos lleva a la necesidad de que las instituciones formales de enseñanza y aprendizaje promuevan "aprendizajes profundos" (Esteban-Guitart y Gee, 2020), con "sentido y valor personal" (Coll, Esteban-Guitart y Iglesias, 2020), también con impacto-beneficio-contribución social. Un aprendizaje profundo es aquél en que el aprendiz participa activamente en una actividad o práctica sociocultural (hacer), construye saberes y destrezas (saber) y se apropia y construye identidades específicas (ser) (Gee y Esteban-Guitart, 2019). Dicho con otras palabras, en el aprendizaje profundo participan las manos (la acción), el corazón (la pasión, el interés, la implicación, la motivación, los afectos y emociones) y la razón (conocimientos, saberes, destrezas) hacia procesos de transformación y cambio, tanto a nivel personal (por ejemplo, poryectando proyectos de vida saludables) como social (incidiendo competencialmente en la comprensión y transformación del mundo). Normalmente, el contexto formal como la escuela y universidades solo activan la razón-cognición, con tareas normalmente descontextualizadas y alejadas de problemas reales, locales y globales, de la vida cotidiana. Estos aprendizajes profundos tienen sentido y valor personal pues parten de nuestras necesidades, intereses y decisiones, permitiéndonos desarrollar un rol activo en la actividad, así como, contribuyendo a una mejor comprensión de nuestro pasado y presente, y facilitando la elaboración de expectativas y planos de actuación futura. Además, se espera que dichos aprendizajes sean beneficiosos socialmente, tengan un impacto social positivo: contribuyan en prácticas encaminadas a los Objetivos de Desarrollo Sostenible, por ejemplo. Disponemos de una gran cantidad de recursos, experiencias y estrategias de personalización educativa, aún hoy minoritarias en la práctica educativa escolar y universitaria, que en ningún caso deben entenderse como estrategias de individualización del aprendizaje, ni prácticas neoliberales aplicadas en el ámbito educativo, sino más bien andamiajes sociales que contribuyan al desarrollo de trayectorias personales de aprendizaje capacitadoras y enriquecidas (Coll, EstebanGuitart, Iglesias, 2020).

Tiempos y espacios educativos expandidos e interconectados. La construcción de trayectorias personales de aprendizaje capacitadoras que vinculen los intereses, necesidades y proyectos identitarios de las personas con las oportunidades educativas, online y offline, constituyen el foco de la actuación educativa en la que cabe situar en el centro al aprendiz, no la práctica homogénea y homogeneizadora de la escuela. Contrariamente al sesgo formal en los procesos de enseñanza y aprendizaje, descrito anteriormente, el aprendizaje aparece como necesariamente, hoy más que nunca, a lo largo y también a lo ancho, de nuestras vidas (Esteban-Guitart, Coll y Penuel, 2018). A 
lo largo debido a la caducidad de los saberes y destrezas, en permanente proceso de actualización, así como a un mercado laboral precario y temporal que nos obliga a (re)inventar nuestras capacidades y conocimientos. Por ello, la oferta educativa desde el contexto no formal se define como extensa, variada y ofrece enormes posibilidades de aprendizaje. No obstante, la conexión de sus tiempos y espacios ofrece más garantías ante el reto ineludible de la equidad e igualdad de oportunidades para su acceso, algo que sin duda condicionará la educación post-Covid-19. A lo ancho debido a la importancia de prácticas, contextos y situaciones informales de vida que nos ofrecen aprendizajes con sentido y valor personal, dónde la intencionalidad educativa es invisible, tácita, incrustada en la actividad (procesos de "educación invisible" en el nicho familiar, con el grupo de iguales, a través de los medios digitales-Internet, videojuegos-, o a través de grupos de afinidad en la web social) así como, recursos, servicios y agentes comunitarios varios (culturales, educativos, sociales, etc.), (Esteban-Guitart, Vila y Serra, 2017; Vila y Esteban-Guitart, 2017). A pesar de no ser una idea nueva (Bronfenbrenner, 1976; Carpenter y McLuhan, 1968), la noción de tiempos y espacios educativos expandidos nos remite a (re)considerar tanto la naturaleza del foco de la actuación educativa (trayectorias personales de aprendizaje con contribución e impacto social), así como, los tiempos y espacios educativos, hoy distribuidos y potencialmente múltiples (EstebanGuitart, Monreal-Bosch, Palma y González-Ceballos, 2020). En esta ecología educativa la escuela está llamada a un importante protagonismo en tanto que agente que permita desarrollar el "oficio de aprender", así como el enriquecimiento de trayectorias personales de aprendizaje; conjuntamente con redes-alianzas educativas comunitarias desde la participación de los ayuntamientos y poderes políticos, así como entidades y agentes comunitarios. No en vano otros agentes (por ejemplo, familias) o medios (el caso de internet) se consideran positivamente como recursos pedagógicos susceptibles de contribuir al desarrollo de las personas. Sin embargo, que el aprendizaje se encuentre distribuido, a lo largo y ancho de la vida, no significa que esté (inter)conectado: para ello es necesario identificar, reflexionar y trabajar sobre los intereses, identidades y experiencias de aprendizaje de los aprendices, a nivel micro, así como generar continuidades educativas entre los distintos agentes, contextos y prácticas comunitarias, a nivel meso (Brofenbrenner, 1976).

Por lo tanto, proponemos aquí dos principios para la mejora y transformación educativa, a saber:

- Diseñar prácticas educativas que fomenten procesos de aprendizaje profundo, con sentido y valor personal, y con impacto-contribución social.

- Diseñar prácticas educativas que reconozcan, legitimen y utilicen los recursos, saberes, destrezas de los distintos agentes comunitarios en proyectos compartidos hacía la creación de continuidades educativas.

\section{A modo de conclusión provisional, ¿cómo articular y llevar a cabo la mejora y transformación educativa?}

La (re)consideración del sentido (qué es el aprendizaje), así como la ontología y axiología de la práctica educativa, deben contribuir a la mejora y transformación educativa hacia el empoderamiento y la construcción de personas críticas que no solamente puedan comprender los artefactos e instrumentos psicológicos contemporáneos (los medios 
digitales, por ejemplo) sino sobre todo permitan el desarrollo y supervivencia de nuestra especie. Un cambio de paradigma, reivindicado hoy por distintos pensadores y sociólogos contemporáneos (por ejemplo, Castells, 2020), requiere -entre muchos otros cambios- de un examen crítico de las ciencias del aprendizaje y de la educación.

A partir de los sesgos y principios descritos anteriormente, y como conclusión final, sugerimos algunas consecuencias para la intervención y actuación educativa, ilustradas con distintos ejemplos empírico-reales. De modo que la mejora y transformación educativa se articule considerando los siguientes planteamientos:

Educación crítica que permita comprender la gramática de los lenguajes contemporáneos, así como socialmente comprometida a partir de la involucración en la búsqueda de soluciones y alternativas a cuestiones, problemas y fenómenos transversales. Por ejemplo, abordando cuestiones como: ¿Por qué al poner una palabra en un buscador hay una página-recurso-enlace que aparece primero?, ¿dónde se guardan nuestras "huellas digitales" y quién tiene acceso a ellas?, ¿qué riesgos y oportunidades se derivan de los videojuegos?, ¿qué relación existe entre objetividad y medios de comunicación?, ¿cómo habitar internet y las redes más allá del mero acceso a contenidos o canal de comunicación con otros, para entenderlo como contexto y práctica de aprendizaje?, ¿cómo se dan los procesos de discriminación y racismo?, ¿cómo identificarlos? Con el objetivo de fomentar alfabetizaciones críticas se requiere que el aprendiz adopte una actitud activa y, por ende, que la práctica educativa gire alrededor de él o ella, no del docente, la asignatura o de un contenido memorístico determinado. Por ejemplo, en propuestas basadas en el aprendizaje-servicio, los alumnos y alumnas realizan aprendizajes competenciales a través de dar respuesta a necesidades y demandas reales de su entorno comunitario (García-Romero y Lalueza, 2019). En el caso del Phenomen Based Learning.3, implementado en Finlandia a partir del año 2016, los estudiantes exploran identidades en tanto que matemáticos, investigadores, exploradores, evaluadores, a partir del abordaje pluridisciplinar frente a un determinado fenómeno. Por ejemplo, el fenómeno del agua permite tratar temas de química, sostenibilidad, economía, turismo o literatura; o la Unión Europea permite trabajar idiomas, geografía o historia. ¿Por qué no convertir el Covid-19 como fenómeno a través del cual trabajar competencias digitales, competencias matemáticas, lingüísticas, así como conocimientos generales útiles para la vida diaria como ciudadano para entender los medios de comunicación, los avances de la ciencia, o los procesos de decisión y actuación política? Se trata, en definitiva, de convertir la realidad cotidiana en objeto y vehículo curricular a partir de su problematización-interrogación con fines pedagógicos. Otro ejemplo ilustrativo es el modelo FUSEstudio ${ }^{4}$ a propósito de retos, que los aprendices eligen en función de sus intereses, y que promueven competencias vinculadas a ciencias, tecnología, ingeniería, artes y matemáticas como, por ejemplo, diseñar la casa de tus sueños, crear e imprimir una versión de uno/a mismo/a con tecnología $3 \mathrm{D}$, o aprovechar el viento para convertirlo en electricidad (DiGiacomo, Van Horne y Penuel, 2020). Por otra parte, en el caso de los Estados Unidos de América, se han diseñado currículums basados en la indagación y la vinculación con cuestiones de importancia comunitaria. Por ejemplo, currículums en biología basados, en lugar de en clases

\footnotetext{
${ }^{3}$ http://www.phenomenaleducation.info/phenomenon-based-learning.html

${ }^{4}$ https://www.fusestudio.net/challenges
} 
convencionales, en el trabajo autónomo y colaborativo de los estudiantes frente a cuestiones como “¿qué explica que la población de búfalos en Tanzania (África) haya cambiado tanto?”, así como en alianzas a largo plazo, basadas en procesos de co-diseño entre docentes e investigadores, para analizar y solventar problemas derivados de la práctica como, por ejemplo, redistribuir las oportunidades educativas en clases de ciencias ("equidad"), así como reparar y sostener percepciones de los estudiantes en tanto que agentes capaces de saber y razonar en clases de ciencias, y en el contexto de su comunidad (“justicia epistémica”) (Penuel y Watkins, 2019).

Educación de los afectos y los cuidados no tanto a partir de una asignatura particular sobre las atenciones o la solidaridad, sino a partir de su desarrollo y práctica. Lo que Bronfebrenner (1978) llamó currículum para el cuidado (curriculum for caring): niños y niñas asumiendo responsabilidades y ofreciendo atención, bajo la supervisión de adultos, con criaturas más pequeñas, o personas mayores. Se trata de actuaciones basadas en el cuidado, que ponen el acompañamiento, la escucha activa y la empatía, en el centro de la actividad. En este sentido, las escuelas, institutos y universidades, concebidas tradicionalmente como comunidades de aprendizaje, debieran también reconocerse como comunidades de los cuidados y los afectos en lo que Hargreaves (2001) llamaba geografías emocionales de la enseñanza o Rajala y Lipponen (2018), en el contexto finlandés, llaman culturas de la compasión. En definitiva, la enseñanza y el aprendizaje se puede entender como una forma necesaria, y especial, de intercambio, cuidado y cooperación mutua. Estamos, en este sentido, completamente de acuerdo con Tarabini (2020) cuando recientemente reivindicaba, recuperando a Freire, la necesidad de "una figura docente capaz de acompañar a su alumnado en el sentido más amplio de la palabra. Acompañar significa estar o ir en compañía del otro, existir junto al otro, participar en sus sentimientos" (p. 152). Para ello nos parece indispensable fomentar procesos de (re)conocimiento empírico de las biografías personales y comunitarias de los aprendices -en línea con la pedagogía del acompañamiento de Sepúlveda (2011)-, así como promover el intercambio activo y establecimiento de relaciones basadas en la confianza mutua entre docentes-estudiantes, docentes-familias, docentes-agentesservicios comunitarios. Por ejemplo, en la aproximación de los fondos de conocimiento, anteriormente mencionada, los docentes visitan los hogares de sus alumnos con el objetivo de (re)presentar sus familias y comunidades en base a sus fortalezas, recursos, saberes y competencias, y (re)orientar la práctica educativa hacia modelos más contextualizados y culturalmente congruentes, facilitando el establecimiento de unas mejoras relaciones entre las familias y el contexto escolar (González y Moll, 2002; Llopart y Esteban-Guitart, 2018; Moll, 2019). Precisamente, a propósito de la pandemia actual, asistimos a nuevas maneras potenciales -a través de conexiones, asistencia y acompañamiento mediado digitalmente- de reforzar los vínculos, así como conocimientos mutuos, entre los docentes y las familias. Estimamos necesario consolidar estrategias que permitan reforzar la actuación educativa a través del intercambio y (re)conocimiento mutuo -tanto de la familia por parte del docente, como de la práctica educativa-escolar por parte de la familia-. Lo que facilita, potencialmente, procesos de contextualización y vinculación pedagógica, así como la creación de continuidades educativas entre distintos contextos de vida y socialización (Bronfenbrenner, 1976). También, nos sentimos plenamente reflejados en la noción de "esperanza pedagógica", propuesta por Van Manen (2003, 2004), como condición, significado y experiencia central en los aprendizajes, así como la reivindicación de un compromiso personal por 
parte de educadores y educadoras. Por todo ello, consideramos que los programas de mentoría y acompañamiento permiten trabajar competencias de sensibilidad social, ayuda, colaboración y compromiso como, por ejemplo, el "proyecto Rossinyol" 5 en el que estudiantes de la universidad de distintas edades se convierten en "mentores" de adolescentes y jóvenes de origen extranjero de distintas escuelas e institutos para favorecer la cohesión e inclusión social, el desarrollo lingüístico, así como la vinculación con el territorio. Otro ejemplo lo encontramos con el "proyecto Prometeus" en el que estudiantes de secundaria de barrios específicos de Barcelona, son acompañados desde la tutoría y mentoría para la transición y experiencia universitaria (Alegría et al., 2017). Desde esta iniciativa y a nivel local, participan tanto la administración educativa y municipal, como centros educativos y organizaciones de la comunidad. A su vez y ubicadas fuera de su territorio de origen, participan también distintas universidades. Para su éxito, dichos proyectos se basan en la creación de vínculos afectivos de apoyo, empatía, intercambio y colaboración.

Educación intergeneracional. Las escuelas, institutos y universidades no funcionan, realmente, como espacios de encuentro e intercambio intergeneracional. De hecho, existe una clara segregación por edad. Se requiere, en este sentido, de prácticas en las que exista intercambio y actividades conjuntas inter-edades. Por ejemplo, el programa "Cosas de la edad" ${ }^{6}$ documentó a lo largo de 6 semanas el progreso de un grupo de niños y niñas de 4 años de edad en una residencia de ancianos para compartir tiempo, juegos, emociones, experiencias y actividades varias. Reivindicamos, en este sentido, la educación intergeneracional como más necesaria que nunca. La "educación post-Covid19” requerirá del fortalecimiento de vínculos interpersonales entre miembros de una misma comunidad. La distancia social experimentada estas semanas nos plantea la necesidad de retomar las relaciones personales y físicas como base pedagógica y valor colectivo, ante unos procesos de construcción del aprendizaje que cada día evidencian mayor base social.

Educación personalizada. Recoge un conjunto diverso y rico de estrategias y actuaciones encaminadas a favorecer procesos de aprendizaje con sentido y valor personal por parte de los aprendices. Se distinguen entre estrategias de contextualización educativa a partir de la identificación, reflexión y trabajo sobre los intereses, necesidades, identidades y decisiones de los alumnos y alumnas; así como vinculadas a la organización de los tiempos, espacios y agentes educativos (Coll, Esteban-Guitart y Iglesias, 2020). La conexión de textos con experiencias personales, estudios empíricos sobre los intereses y comunidades de referencia de los estudiantes, la utilización de sus prácticas y referentes de sentido (por ejemplo, la música), o la conexión de sus intereses con los servicios y oportunidades de la comunidad son actuaciones que facilitan procesos de aprendizaje con sentido. Por ejemplo, Versembrant ${ }^{7}$ es una escuela itinerante que utiliza el arte urbano y el hip hop como instrumento para fomentar la consciencia crítica. También en el contexto de Catalunya, el SINS Cardener se organiza alrededor de "planes personales de aprendizaje” a través de los cuales los estudiantes, conjuntamente con sus docentes-

\footnotetext{
${ }^{5}$ http://www.projecterossinyol.org/?lang=es

$6 \quad$ https://www.youtube.com/watch?v=o4CC1fdNc7U\&list=PLSXGfg6XHVB4Na-

BA7KQnICX2yyWooTeS

${ }^{7}$ https://www.versembrant.cat
} 
tutores, y sus familias, deciden y consensuan los objetivos de aprendizaje que les llevan a desarrollar -a lo largo del curso- itinerarios particulares de entre distintos proyectos y actividades curriculares disponibles (Atzet et al., 2018). Ello rompe la rigidez y homogeneidad de los tiempos y actividades educativas convencionales, hacia modelos más flexibles que incorporen la voz, responsabilidad y participación de los y las aprendices en los procesos de decisión sobre el qué, cómo, cuándo, con quién aprender, y cómo evaluar lo aprendido, para seguir revisando los objetivos de aprendizaje establecidos.

Educación compartida y conectada. Se trata de alianzas entre distintas entidades y agentes que rompen con las fronteras y muros tradiciones de la escuela. Dichas prácticas híbridas incorporan agentes, contextos, recursos y servicios de la comunidad bajo marcos de intervención educativa con objetivos y actividades comunes. El espacio público digital es la aportación genuinamente nueva de la revolución de base tecnológica que está teniendo lugar en este momento histórico pre y post Covid-19. Los medios digitales sociales han provocado una ingente cantidad de intervenciones educativas (forzadas o espontáneas como resultado del ánimo de innovar) que a su vez han estimulado la investigación para interpretar la naturaleza de los cambios y poder convertirlos en progreso. Una cantidad creciente de autores llaman la atención sobre la necesidad de ayudar a comprender cómo han afectado las nuevas formas de aprender, participar o de organizarnos en la institución educativa, sin exagerar ni las expectativas de transformación de la tecnología ni los peligros de la exposición a unos medios de difusión potencialmente ilimitada. En este sentido la pretensión de nuestras propuestas es ayudar a construir un conocimiento suficientemente crítico como para intervenir e innovar en aquellos procesos de cambio y mejora, entendiendo las lógicas de lo digital como una forma de estar y dar sentido al mundo en el que vivimos (González-Patiño,

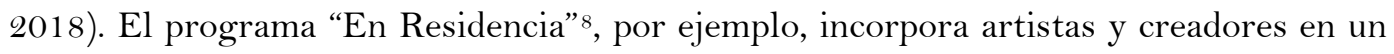
curso escolar que realizan una obra conjuntamente con estudiantes de entre 12 y 16 años de edad. La escuela Dr. Robert, otro ejemplo, ha incorporado en su proyecto educativo, en particular en el consejo escolar ${ }^{9}$, no solamente los docentes del centro, sino también las familias, estudiantes, y entidades de la comunidad (como los ayuntamientos colindantes, así como asociaciones y fundaciones) con el fin de crear una comunidad educativa más amplia. Se trata de actuaciones que tienden a crear puentes de colaboración, diagnóstico y actuación compartida, entre las distintas entidades sociales y educativas de un determinado territorio. En este sentido, en el año 2018 se creó la Alianza "Educación 360"10 en Catalunya, promovida por la Fundación Bofill, la Federación de Movimientos de Renovación Pedagógica de Catalunya, así como la Diputación de Barcelona. En la actualidad, año 2020, la alianza cuenta con cerca de 300 socios: entidades locales como ayuntamientos, centros escolares, grupos de investigación y universidades, así como entidades o redes profesionales varias vinculadas al deporte y actividad física, las artes, o la educación en el tiempo libre. El propósito es mejorar la equidad educativa a través de la conexión de aprendizajes, tiempos, espacios y agentes educativos. Con ello, se persigue crear ecosistemas educativos comunitarios, es decir, una red de relaciones basadas en el reconocimiento y confianza mutua entre distintas

\footnotetext{
8 https://www.enresidencia.org/es

${ }^{9}$ https://en.calameo.com/read/00250353091d7a27e1828?page=2 1

${ }^{10}$ https://www.educacio360.cat
} 
entidades, recursos y agentes sociales y educativos; bajo una agenda, diagnóstico, objetivos compartidos y reales, y mediante un trabajo horizontal constante, participativo en busca de la materialización de la finalidad anteriormente anunciada. Por ejemplo, en el municipio de Celrà (Girona, Catalunya), se creó una alianza entre los centros educativos, las familias, técnicos y concejales del ayuntamiento, así como entidades y equipamientos como la Escuela Municipal de Danza. Se identificaron colectivamente, a través de métodos participativos, cuatro retos educativos (por ejemplo, mejorar el conocimiento entre los distintos agentes y servicios del municipio, así como la coordinación entre las distintas entidades y agentes profesionales). A partir del diagnóstico compartido, se establecieron distintas actuaciones como la creación de un espacio web "Portal 360 " que identifique y socialice las distintas asociaciones, entidades, agentes y actuaciones educativas y comunitarias del territorio; las "familias mentoras" para acompañar a las personas que se incorporan al municipio; o consensuar un "tema transversal” para organizar una formación que permita establecer continuidades entre los distintos agentes y entidades. Por ejemplo, cómo incorporar la perspectiva de género, o hacer frente a situaciones de racismo y discriminación, tanto en las políticas municipales, la escuela de Danza, las entidades del tiempo libre o las instituciones formales de enseñanza y aprendizaje. Nos parece, en el contexto de la educación postCovid-19, que las propuestas educativas venideras deberán dar respuesta a las necesidades y desafíos que a nivel local afronte cada comunidad educativa. Siempre, a partir del (re)conocimiento de los distintos servicios, agentes, equipamientos y recursos educativos-comunitarios; reforzando la complementariedad, funciones y conocimientos entre ellos, así como la especificidad y singularidad en sus funciones y competencias. No obstante, conviene recordar que en tiempos de crisis como el actual existe y existirá la tentación a una reacción de cierre en las organizaciones educativas y comunitarias. Es decir, fundamentadas en base al propósito legítimo de conservar aquello que se considera suyo. Cualquier organismo frente a una amenaza externa (pensemos en las dificultades económicas que vendrán para sostener y financiar proyectos educativos) puede reaccionar instintivamente asegurando aquello que tiene. En cambio, la educación postCovid-19 apuesta por la configuración de nodos y mecanismos para articular redes de aprendizaje desde un doble propósito: para generar más oportunidades de aprendizaje y también, no menos importante, porqué es una manera eficiente de garantizar que cada organización educativa conserve aquello que considera suyo, desde el valor de lo comunitario.

Educación para la sostenibilidad ambiental, identitaria y cultural. La escuela, como proyecto democrático, inclusivo y sostenible, debe contribuir a la comprensión y sensibilidad frente al colapso ecológico (calentamiento global, pérdida de la biodiversidad, cambio climático), así como la multi-pertenencia identitaria que permita reconocer los lazos y vínculos como especie, y con ella, una identidad planetaria, más allá de lenguas, identidades culturales e ideologías, pero también circunscrita a la riqueza y diversidad social y cultural de las comunidades de referencia (Esteban-Guitart et al, 2020). De hecho, no solamente debe ser culturalmente congruente, reconociendo las culturas y nacionalidades de los aprendices, sino también garantizar la supervivencia de dichas prácticas, códigos y registros culturales. En este sentido, una educación culturalmente sostenible debe normalizar la diferencia y diversificar la normalidad (ofrecer espacios para el aprendizaje de lenguas infra-representadas en el espacio escolar, o para el aprendizaje de la lengua de signos, por ejemplo, como actividad lectiva). Por 
ejemplo, la experiencia "Ciberbressol" en la que un grupo de jóvenes gitanas crearon en la Asociación Gitana de Badalona un espacio en el que jóvenes madres del barrio se encontraban con sus hijos en un entorno híbrido, con juguetes y estoras en el suelo, y ordenadores en las mesas, desarrollando una actividad de juego para los niños y de intercambio para las madres, al tiempo que éstas participaban en un curso orientado a la obtención del graduado en ESO. Es decir, ludoteca, escuela de familias y escuela de segunda oportunidad en un espacio híbrido culturalmente definido por prácticas propias de una comunidad gitana y prácticas de educación formal (Lalueza, Sánchez-Busqués y García-Romero, 2019). En este sentido, la aproximación de los fondos de conocimiento e identidad, anteriormente mencionada, persigue vincular el currículum y objetivos pedagógicos con las vidas de los estudiantes y sus familias dentro y fuera de los tiempos y espacios escolares (Esteban-Guitart, 2016). Desde la perspectiva de reconocimiento que señalamos, se considera que todas las familias, en todas las circunstancias, disponen de experiencias vitales, y con ellas, acumulan y construyen saberes, habilidades, recursos y conocimientos. El reto pedagógico consiste en identificarlos empíricamente. Para ello, como ya se ha dicho anteriormente, los docentes visitan los hogares de sus estudiantes y documentan los recursos, destrezas y saberes que éstas tienen, para posteriormente establecer conexiones con el currículum y la práctica escolar. Por ejemplo, tras identificar los cacahuetes como planta para cocinar en distintas familias visitadas de origen marroquí en Catalunya, las docentes diseñaron una actividad pedagógica para trabajar el ciclo vital de las plantas a propósito de la planta de cacahuete. Las familias participaron en la actividad, y además se realizó un diccionario multilingüe para incorporar las distintas lenguas maternas de los y las estudiantes y sus respectivas familias (Esteban-Guitart et al., 2019).

En definitiva, la naturaleza indefinida de lo humano nos obliga a un constante ejercicio de mejora y transformación, tanto a nivel personal, como social. Sucesos históricos críticos de inflexión, como la pandemia Covid-19, nos advierte de nuestras fragilidades, nuestros defectos y debilidades; pero también, humildemente, de nuestras fortalezas y potencialidades. Reforzarlas es lo mínimo que se espera de nosotros y nosotras. Una responsabilidad que debe tejerse desde nuestras manos (acciones), nuestras cabezas (inteligencia, ideas, razones) y nuestros corazones (sentimientos, anhelos, emociones).

\section{Referencias}

Alegría, J., Esclapés, E., Iglesias, E., Quintela, J. y Lleonart, P. (2017). Proyecto prometeus: Acceso y permanencia en la universidad. En M. El Homrani, F. Peñafiel, y A. Hernández (Eds.), Entornos y estrategias educativas para la inclusión social (pp. 143-156). Editorial Comares.

Álvarez, M., Gardyn, N., Iardelevsky, A. y Rebello, G. (2020). Segregación educativa en tiempos de pandemia: balance de las acciones iniciales durante el aislamiento social por el covid-19 en Argentina. Revista Internacional de Educación para la Justicia Social, 9(3), 25-43. https://doi.org/10.15366/riejs2020.9.3.002

Atzet, D., Cara, Y., Martínez Garcia, A., Rodríguez, M., López Roca, I. (2018). L’organtizació de l'acció educativa a partir dels plans personals d'aprenentatge. Dossier Graó, 3, 34-37.

Bauman, Z. (2006). Liquid times: Living in an age of uncertainity. Polity Press. 
Belavi, G. y Murillo, F. J. (2020). Democracia y justicia social en las escuelas: Dimensiones para pensar y mejorar la práctica educativa. REICE. Revista Iberoamericana sobre Calidad, Eficacia y Cambio en Educación, 18(3), 5-28. https://doi.org/10.15366/reice2020.18.3.001

Blundell, A. (2020). The coronavirus pandemic is a world perezhivanie. Cultural Praxis. http://culturalpraxis.net/wordpress 1/2020/04/15/the-coronavirus-pandemic-is-aworld-perezhivanie/

Bozkurt, A. y Sharma, R. C. (2020). Emergency remote teaching in a time of global crisis due to coronavirus pandemic. Asian Journal of Distance Education, 15(1), 1-6. https://doi.org/10.5281/zenodo.3778083

Bronfenbrenner, U. (1976). The experimental ecology of education. Educational Researcher, 5(9), 5-15. https://doi.org/10.2307/1174755

Bronfenbrenner, U. (1978). Who needs parent education? Teachers College Record, 79(4), 767-787.

Brühlmeier, A. (2012). Cabeza, corazón y mano. Educando al ser humano en el espíritu de Pestalozzi. Shule für das Kind.

Cabrera, L. (2020). Efectos del coronavirus en el sistema de enseñanza: aumenta la desigualdad de oportunidades educativas en España. Revista de Sociología de la Educación-RASE, 13(2), 114-139. https://doi.org/10.7203/RASE.13.2.17125

Carbonell, E. (13 de abril de 2020). La Covid-19 es el último aviso y, sin conciencia crítica de especie, a la próxima la humanidad colapsará. Público.

https://www.publico.es/entrevistas/entrevista-eudald-carbonell-covid-19-avisoconciencia-critica-especie-proxima-humanidad-colapsara.html

Carpenter, E. y McLuhan, M. (1968). El aula sin muros. Investigaciones sobre técnicas de comunicación. Ediciones de Cultura Popular.

Castells, M. (4 de abril de 2020). Fin de un mundo. La Vanguardia. https://www.lavanguardia.com/opinion/20200404/48287445172/fin-de-un-mundo.html

Cervantes Holguín, E. y Gutiérrez Sandoval, P. R. (2020). Resistir la Covid-19. Intersecciones en la educación de Ciudad Juárez, México. Revista Internacional de Educación para la Justicia Social, 9(3), 7-23. https://doi.org/1015366/riejs2020.9.3.001

Coll, C. (2013). El currículo escolar en el marco de la nueva ecología del aprendizaje. Aula de Innovación Educativa, 219, 31-36.

Coll, C., Esteban-Guitart, M. y Iglesias, E. (2020). Aprenentatge amb sentit $i$ valor personal. Experièncias, recursos $i$ estratègies de personalització educativa. GRAO.

Collado-Ruano, J. (2016). Epistemologías del sur: Una visión descolonial a los objetivos de desarrollo sostenible. Sankofa, 9, 137-158. https://doi.org/10.11606/issn.19836023.sank.2016.119065

DiGiacomo, D., Van Horne, K. y Penuel, W. (2020). Choice and interest in designed learning enviroments: The case of FUSE Studios. Information and Learning Sciences, 9, 98-107. https://doi.org/10.1108/ILS-09-2019-0098

Engeström, Y. (2001). Expansive learning at work: Toward an activity theoretical reconceptualization. Journal of Education and Work, 14(1), 136-156.

Esteban-Guitart, M. (2016). Funds of identity. Connecting meaningful learning experiences in and out of school. Cambridge University Press. 
Esteban-Guitart, M. y Gee, J. (2020). Inside the head and out in the world. An approach to Deep teaching and learning. REMIE. Multidisicplinary Journal of Educational Research, 10, 1-25. https://doi.org/10.17583/remie.2020.4868

Esteban-Guitart, M., Coll, C. y Penuel, W. (2018). Learning across settings and time in the digital age. Digital Education Review, 33, 1-16. https://doi.org/10.1344/der.2018.\%25p

Esteban-Guitart, M., Serra, J. M. y Vila, I. (2017). Informationalism and informalization of learning in 21 st century. A qualitative study on meaningful learning experiences. Social and Education History, 6(1), 1-25. https://doi.org/10.17583/hse.2017.2111

Esteban-Guitart, M., Lalueza, J. L., Zhang-Yu, C. y Llopart, M. (2019). Sustaining students' cultures and identities. A qualitative study based on the funds of knowledge and identity approaches. Sustainability, 11(12), 3400. https://doi.org/10.3390/su11123400

Esteban-Guitart, M., Monreal-Bosch, P., Palma, M. y González-Ceballos, I. (2020). Sustaining students' identities within the context of participatory culture: Designing, implementing and evaluating an interactive learning activity. Sustainability, 12(12), 4870.

https://doi.org/10.3390/su12124870

García-Romero, D. y Lalueza, J. L. (2019). Procesos de aprendizaje en aprendizaje-servicio universitario: Una revisión teórica. Educación XX1, 22(2), 45-68.

https://doi.org/10.5944/educXX1.22716

Gee, J. y Esteban-Guitart, M. (2019). Designing for deep learning in the context of digital and social media. Comunicar, 58, 9-18. https://doi.org/10.3916/C58-2019-01

Giroux, H. (2015). Pedagogías disruptivas y el desarrollo de la justicia social bajo regímenes neoliberales. Revista Internacional de Educación para la Justicia Social, 4(2), 13-27. https://doi.org/10.15366/riejs2015.4.2

González, N. y Moll, L. (2002). Cruzando el puente: Building bridges to funds of knowledge. Educational Policy, 16(4), 623-641. https://doi.org/10.1177/0895904802016004009

González-Patiño, J. (2018). Learn, teach and share, participation in expanded educational communities: Seminar as a practice of continuing education between childhood professionals. Digital Education Review, 33, 203-216.

https://doi.org/10.1344/der.2018.33.203-216

González-Patiño, J. y Esteban-Guitart, M. (2014). Some of the challenges and experiences of formal education in a mobile-centric society. Digital Education Review, 25, 64-86. https://doi.org/10.1344/der.2014.25.64-86

Hargreaves, A. (2001). Emotional geographies of teaching. Teachers College Record, 103(6), 10561080.

Ishimaru, A. M. (11 de Mayo de 2020). The prospects for just schools in the wake of covid-19 responses. https://www.tcpress.com/blog/prospects-schools-wake-covid-19-responses/

Jenkins, H., Peters-Lazaro, G. y Shresthova, S. (2020). Popular culture and the civic imagination. Case studies of creative social change. New York University Press.

Lalueza, J.L., Sánchez-Busqués, S. y García-Romero, D. (2019). Following the trail of the 5th dimension: University-community partnership to design educational environments. Mind, Culture \& Activity, 16, 59-78. https://doi.org/10.1080/10749039.2019.1611859

Levitas, R. (2013). Utopia as method: The imaginary reconstitution of society. Springer.

Lingard, B. y Keddie, A. (2013). Redistribution, recognition and representation: Working against pedagogies of indifference. Pedagogy, Culture $\mathcal{E}^{2}$ Society, 21(3), 427-447.

https://doi.org/10.1080/14681366.2013.809373 
Llopart, M. y Esteban-Guitart, M. (2018). Funds of knowledge in 21st century societies: inclusive educational practices for under-represented students. A literatura review. Journal of Curriculum Studies, 5o(2), 145-161. https://doi.org/10.1080/00220272.2016.1247913

Moll, L. (2019). Elaborating funds of knowledge: Community-oriented practices in international contexts. Literacy Research: Theory, Method, and Practice, 68(1), 130-138. https://doi.org/10.1177/2381336919870805

Murillo, F. J. y Duk, C. (2020). El Covid-19 y las brechas educativas. Revista Latinoamericana de Educación Inclusiva, 14(1), 11-13. https://doi.org/10.4067/So718-7378020000100011

Murillo, F. J. y Hernández, R. (2014). Liderando escuelas Justas para la justicia social. Revista International de Educación para la Justicia Social, 3(2), 13-32.

Nasir, N. S., Lee, C. D., Pea, R., Royston, M. M. (2020). Handbook of the cultural foundations of learning. Routledge.

Penuel, W. y Watkins, D. (2019). Assessment to promote equity and epistemic justice: A use-case of a research-practice partnership in science education. The ANNALS of the American Academy of Political and Social Science, 683(1), 201-216. https://doi.org/10.1177/000271621984349

Poveda, D. (2001). La educación de las minorías étnicas desde el marco de las continuidadesdiscontinuidades familia-escuela. Gazeta de Antropología, 17, 1-17.

Rajala, A. y Lipponen, L. (2018). Early childhood education and care in Finland: Compassion in narrations of early childhood education student teachers. En S. Garvis, S. Phillipson y H. Harju-Luukkainen (Eds.), International perspectives on early childhood education and care: early childhood education in the 21 st century (pp. 64-75). Routledge.

Richmond, W. K. (2018). Education and schooling. Routledge.

Rodríguez-Romero, M. (2020). Investigación educativa, neoliberalismo y crisis ecosocial. Del extractivismo a la reciprocidad profunda. REICE. Revista Iberoamericana sobre Calidad, Eficacia y Cambio en Educación, 18(2), 135-149. https://doi.org/10.15366/reice2020.18.2.007

Rodríguez-Ruiz, B., Álvarez-Blanco, L., Martínez-González, R. A. y Epstein, J. L. (2019). Presentación del número relación entre centros educativos, familias y entidades comunitarias. Aula Abierta, 48(1), 7-10. https://doi.org/10.17811/rifie.48.1.2019

Rogoff, B. (2016). Culture and participation: A paradigm shift. Current Opinion in Psychology, 8, 182-189. https://doi.org/10.1016/j.copsyc.2015.12.002

Ruiz, G. R. (2020). Marcas de la pandemia: El derecho a la educación afectado. Revista Internacional de Educación para la Justicia Social, 9(3), 43-59. https://doi.org/10.15377/riejs2020.9.3.003

Santos, B. D. S. (2018). The end of the cognitive empire: The coming of age of epistemologies of the south. Duke University Press.

Sepúlveda, E. (2011). Toward a pedagogy of acompañamiento: Mexican migrant youth writing from the underside of modernity. Harvard Educational Review, 81(3), 550-573. https://doi.org/10.17763/haer.81.3.088mv5t704828u67

Tarabini, A. (2020). ¿Para qué sirve la escuela? Reflexiones sociológicas en tiempos de pandemia global. Revista de Sociología de la Educación-RASE, 13(2), 145-155. https://doi.org/10.7203/RASE.13.2.17135

Valdés, G. (1996). Con respeto: Bridging the distances between culturally diverse families and schools. Teachers College Press. 
Valencia, R. (2010). Dismantling contemporary deficit thinking: Educational thought and practice. Routledge.

Van Manen, M. (2003). Investigación educativa y experiencia vivida. Idea Books.

Van Manen, M. (2004). El tono en la enseñanza. Paidós.

Vila, I. y Esteban-Guitart, M. (2017). Familia, escuela y comunidad en las sociedades del siglo XXI. Editorial Horsori.

Von Braun, J., Zamagni, S. y Sorondo, M. S. (2020). The moment to see the poor. Science, 368(6488), 213. https://doi.org/10.1126/science.abc2255

\section{Breve CV de los autores}

\section{Edgar Iglesias Vidal}

Doctor en pedagogía, licenciado en antropología social y cultural y diplomado en trabajo social. Es miembro del grupo de investigación LIBERI, en Infancia, Juventud y Comunidad de la Universidad de Girona y es profesor asociado del Departamento de Pedagogía de esta universidad. Ha participado en diferentes investigaciones y publicaciones orientadas al reconocimiento de la diversidad sociocultural y la dimensión intercultural en entornos educativos de diversidad: Educación intercultural en el tiempo libre. Acción educativa en tiempos neoliberales (2015). Sus intereses de investigación se orientan también al desarrollo de entornos de aprendizaje comunitarios desde la conexión de agentes y aprendizajes. Fue en el ámbito de la conexión entre el contexto educativo formal y no formal que realizó su estancia postdoctoral de investigación a la Graduate School of Education de la Stanford University (California - EE.UU.). Cuenta con veinte años de experiencia en el ámbito del trabajo socioeducativo en clave comunitaria y es miembro de la Asociación Educativa Integral del Raval, desde donde ha participado en el impulso de diferentes proyectos de educación comunitaria. Colabora también como formador, con diferentes equipos educativos, instituciones y administraciones educativas. ORCID ID: https://orcid.org/0000-0002-0356-0439. Email: edgar.iglesiasv@udg.edu

\section{Javier González-Patiño}

Profesor de la Facultad de Educación de la UAM y miembro de la Cátedra UNESCO de Educación para la Justicia Social, cuenta con una amplia experiencia como creativo digital y es fundador de Mediática, colectivo que asesora e investiga sobre la transformación digital de comunidades de aprendizaje. La intersección entre trayectoria profesional y académica orienta sus intereses de investigación, tendiendo a implicarse en proyectos participativos en diferentes contextos socio-culturales que permitan promover y analizar la mediación digital en procesos educativos y de comunicación mediante una perspectiva transdisciplinar. Ha realizado numerosas publicaciones sobre relaciones familia escuela, cultura digital y la participación activa de la infancia en procesos educativos. A través de Mediática elabora proyectos con comunidades o colectivos (centros educativos, grupos de investigación, empresas, instituciones, etc.) para que innoven en sus prácticas de creación de conocimiento, explorando y aprovechando las características y dinámicas de la web social como espacio público conectado. Colabora habitualmente en Medialab-Prado Madrid, espacio del Ayuntamiento de Madrid con 
prestigio internacional dedicado a la cultura digital, y participa con frecuencia en actividades de innovación abierta y de transferencia de conocimiento científico. ORCID ID: https://orcid.org/0000-0003-3909-1897. Email: javier.gonzalezpatino@uam.es

\section{José Luis Lalueza}

Doctor en Psicología por la UAB, es profesor en el Departamento de Psicología Básica, Evolutiva y de la Educación de esta universidad. Coordinador del Grupo de Investigación en Desarrollo Humano, Intervención Social e Interculturalidad (DEHISI) ha desarrollado su actividad de Investigación-acción en el ámbito del desarrollo y la construcción de la identidad en comunidades minorizadas y en entornos de exclusión social, en el diseño de entornos de aprendizaje inclusivo y comunidades de prácticas para la educación intercultural, y en estudios y diagnóstico de necesidades en el ámbito del bienestar infantil, la protección de menores y la intervención con familias. Forma parte de la red internacional UClinks, basada en la Universidad de California, en cuyo marco ha contribuido al desarrollo del proyecto educativo "Quinta Dimensión". Está implicado en la promoción del Aprendizaje Servicio (ApS) universitario a través de la ejecución de proyectos docentes, la investigación y la co-coordianción del proceso de institucionalización en la UAB. Es también coordinador del Àrea de Investigación Aplicada y Transferencia del Servicio de Psicología y Logopedia de la UAB. ORCID ID: https://orcid.org/0000-0002-0897-9917. Email: joseluis.lalueza@uab.cat

\section{Moisés Esteban-Guitart}

Licenciado en filosofía y en psicología. Doctor en Psicología. Profesor del departamento de psicología de la Universitat de Girona. Director del Instituto de Investigación Educativa, y responsable del grupo de investigación "Cultura y Educación". Ha sido profesor visitante en la Universidad Intercultural de Chiapas (México), University of Arizona (USA), University of Colorado-Boulder (USA). Su principal línea de investigación es el análisis de procesos de personalización y contextualización educativa (escuela, familia y comunidad); en particular bajo la aproximación de los "Fondos de conocimiento e identidad". Sus libros más recientes son: "Funds of identity. Connecting meaningful learning experiences in and out of school" (2016, New York: Cambridge University Press). "Familia, escuela y comunidad en las sociedades del siglo XXI" (con Ignasi Vila, 2017, Barcelona: Horsori), y "Aprenentatge amb sentit i valor personal. Estratègies, recursos i experiències de personalització educativa" (con César Coll y Edgar Iglesias, 2020, Barcelona: Graó). ORCID ID: https://orcid.org/0000-0002-17008792. Email:moises.esteban@udg.edu 